\title{
A Longterm Prospective Real-life Experience with Leflunomide in Juvenile Idiopathic Arthritis
}

\author{
Antonia Célia de Castro Alcântara, Christiane Araújo Chaves Leite, Ana Caroline \\ Rocha Melo Leite, José Julio Costa Sidrim, Francisco Saraiva Silva Jr., \\ and Francisco Airton Castro Rocha
}

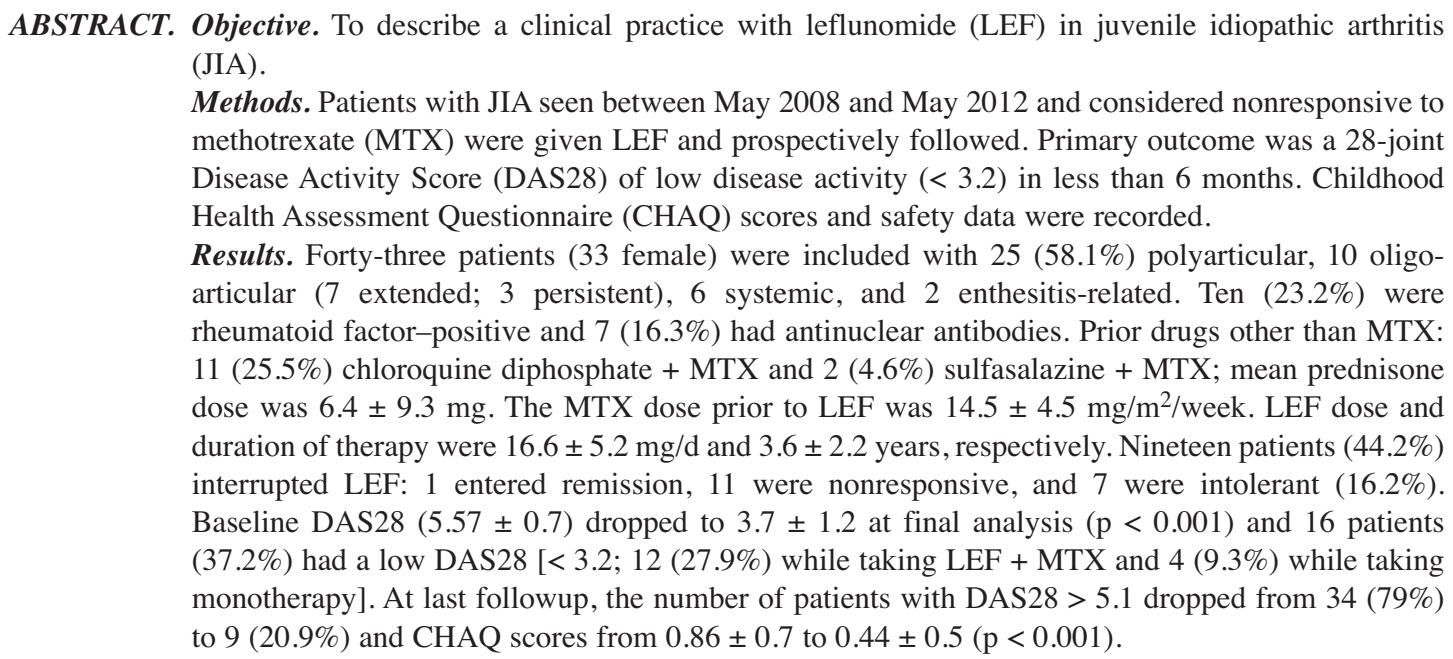

Conclusion. LEF isolated or combined with MTX is effective and safe to treat JIA in patients refractory to MTX. (J Rheumatol First Release Dec 15 2013; doi:10.3899/jrheum.130294)

\section{Key Indexing Terms: JUVENILE IDIOPATHIC ARTHRITIS DISEASE ACTIVITY SCORE}

\section{LEFLUNOMIDE METHOTREXATE DISEASE-MODIFYING ANTIRHEUMATIC DRUGS}

Juvenile idiopathic arthritis (JIA) is the most frequent chronic arthropathy in childhood, affecting 1 or more joints in children less than 16 years old. It is more common among girls. The prevalence of JIA in developing countries has not been extensively studied and differences in the clinical presentation, as well as response to treatment, may vary among patients with JIA worldwide. Methotrexate (MTX) is currently the first choice among disease-modifying antirheumatic drugs (DMARD) in JIA ${ }^{1}$. Although we do not

From the Department of Internal Medicine, Faculty of Medicine, Universidade Federal do Ceará, Fortaleza CE, Brazil.

Supported by grants from CNPq (Conselho Nacional de Desenvolvimento $e$ Tecnológico) and FUNCAP (Fundação Cearense de Apoio ao Desenvolvimento Científico e Tecnológico - PRONEX - 48.01.00/08).

A.C.C. Alcântara, MD, Department of Internal Medicine, Faculty of Medicine; C.A.C. Leite, $M D, P h D$, Department of Pediatrics,

Universidade Federal do Ceará; A.C.R.M. Leite, DDS, PhD, Universidade da Integração Internacional da Lusofonia Afro-Brasileira; J.J.C. Sidrim, MD, PhD, Medical Microbiology Center, Universidade Federal do Ceará; F.S. Silva Jr., MD, PhD; F.A.C. Rocha, MD, PhD, Department of Internal Medicine, Faculty of Medicine, Universidade Federal do Ceará.

Address correspondence to Dr. F.A.C. Rocha, Rua Dr. Jose Lourenço 1930, Aldeota 60115-281, Fortaleza CE, Brazil.E-mail: arocha@ufc.br Accepted for publication October 17, 2013. have written guidelines for the treatment of JIA in Brazil, the current practice among rheumatology centers is also to start with MTX. Leflunomide (LEF) is classified as a DMARD that effectively ameliorates signs and symptoms in patients with rheumatoid arthritis (RA) ${ }^{2}$. In addition, LEF can also be combined to the so-called biologic DMARD to treat patients who have RA that is nonresponsive or who are intolerant to $\mathrm{MTX}^{3}$. In Brazil, a common practice in treating RA is to add LEF to MTX or to use it as monotherapy in those intolerant to MTX ${ }^{4}$. This practice is also followed often in the treatment of JIA. However, concerns persist about less tolerability of LEF because of increased incidence of adverse events (mostly linked to hepatotoxicity) ${ }^{2}$.

Few studies have focused on the efficacy of LEF in JIA. One of those studies compared the administration of either MTX or LEF to patients with JIA. The results showed a mild though significantly better response with MTX for a 16-week period, with acceptable tolerability for both compounds ${ }^{5}$. A retrospective study of children with the polyarticular presentation of JIA who were not responsive to MTX or who could not tolerate it showed that LEF led to clinical improvement in up to $75 \%$ of the patients, including

Personal non-commercial use only. The Journal of Rheumatology Copyright $\odot$ 2014. All rights reserved. 
almost $30 \%$ who achieved remission with an acceptable safety profile 6 .

Clinical presentation as well as response to treatment may vary worldwide among patients with $\mathrm{JIA}^{7}$. Specifically with regard to LEF, there are reports on the possible difference in the prevalence of side effects among various populations, suggesting that the effects are worse in patients from East $\mathrm{Asia}^{2}$. We live in a low latitude region, with a low-income population of about 8.5 million in the northeast of Brazil. Sanitary problems and the hot weather account for a high prevalence of both parasitic and infectious diseases so that helminthic infections are endemic. Therefore, we have special concerns about safety issues owing to opportunistic infections before prescribing biological DMARD for our patients.

Defining specific targets to treatment has been shown to improve outcome in patients with $\mathrm{RA}^{8}$. Criteria for the definition of remission or inactive disease in JIA have been published, but the definition of minimal or low disease activity still needs to be determined ${ }^{9}$. This is particularly important in JIA, because achieving a state of inactive disease has been shown to improve outcome concerning longterm joint damage ${ }^{10}$.

Our aim was to prospectively evaluate the use of LEF in children with JIA who were considered nonresponsive or intolerant to MTX and seen at the outpatient clinics of the Hospital Universitário Walter Cantídio (HUWC) of the Faculty of Medicine of the Universidade Federal do Ceará. Because our study was designed in 2007, we used RA outcomes to evaluate our patients with JIA. To this purpose, low disease activity based on the 28-joint Disease Activity Score (DAS28) definition in RA was the primary outcome to be achieved within a maximum of 6 months ${ }^{11}$.

\section{MATERIALS AND METHODS}

All patients with a diagnosis of JIA according to the International League of Associations for Rheumatology ${ }^{12}$ and seen at the rheumatology outpatient clinics of the HUWC, Fortaleza CE, Brazil, between May 2008 and May 2012 were prospectively evaluated. There were 43 children treated with LEF, with an age range from 1 to 16 years at study entry.

The clinical protocol was submitted and approved by our local Ethics Regulatory Committee, which follows the rules of the Brazilian National Ethics Committee on Clinical Research. All patients or their responsible relatives signed an informed consent form before any intervention. At the start of the protocol, all children were identified after a survey in the pharmaceutical division of the HUWC and had their clinical records evaluated to confirm diagnosis. The fact that LEF is prescribed under a strict regulatory control and is free of charge to the patient guarantees that we had access to all the patients in the period mentioned. We do not have a pediatric rheumatologist in our facility. However, we have been responsible for the care of all children with rheumatologic complaints in our service since 1994. Therefore, all patients were prospectively followed in our unit with the help of a pediatrician.

Our long experience with adult patients with RA has led us to abandon the use of a loading dose of LEF in adults, and we followed the same strategy with children. Dose adjustment for LEF was based on body weight, as follows: patients with $<20 \mathrm{~kg}$ received $10 \mathrm{mg}$ LEF every second day; those between 20 and $40 \mathrm{~kg}$ received $10 \mathrm{mg} \mathrm{LEF} /$ day, and patients weighing more than $40 \mathrm{~kg}$ received $20 \mathrm{mg} \mathrm{LEF} / \mathrm{day}^{5}$. A specific warning document about the malformation risks in case of pregnancy was signed by the parents. When judged necessary by the accompanying pediatrician, the patients were specifically warned of this risk. Those reporting being sexually active received birth control pills after gynecological counseling. A clinical chart was filled for each patient and prospectively evaluated. The data registered included patient demographics, clinical evaluation, current and past treatment, adverse events, and reasons for starting and discontinuing LEF. A complete physical examination was performed and blood sampling was done for routine laboratory tests, including blood cell counts, biochemistry (glucose, creatinine, urea, aspartate aminotransferase, alanine aminotransferase), type I urinalysis, and thorax radiography. Testing for the presence of rheumatoid factor (RF) using nephelometry and antinuclear antibodies using indirect immunofluorescence was also done. Disease activity was analyzed using the DAS28, as developed and used for adult patients with RA, with 4 criteria. The DAS28 measures the number of swollen and tender joints out of a total of 28 joints, in addition to the erythrocyte sedimentation rate (ESR) and general health assessment by the patient (parent) in a $0-100 \mathrm{~mm}$ visual analog scale (VAS) ${ }^{11}$.

Any patient with a DAS28 > 3.2 was considered as having active disease despite MTX use. The Childhood Health Assessment Questionnaire (CHAQ) was used for evaluation of functional status. Any patient taking LEF was included in the intention-to-treat analysis. The main outcome for considering a patient as responsive was achievement of a DAS $28<3.2$ within a maximum period of 6 months after starting LEF. To this purpose, patients entering the protocol were seen monthly by the rheumatologist during the first 6 months. American College of Rheumatology pediatric (ACR-Pedi) responses were also assessed, based on the following variables: ESR; number of joints with active arthritis; number of joints with limited range of motion; physician's global assessment of disease severity (0-100 mm VAS); parents' or child's global assessment of patient's well being (0-100 mm VAS); and CHAQ (0-3). ACR-Pedi30 is defined as an improvement of at least $30 \%$ in 3 of the 6 core criteria with no more than 1 of the criteria worsening by $30 \%$ or more. In ACR-Pedi50, 70, 75, 90, or 100 , improvement had to be at least $50 \%, 70 \%, 75 \%, 90 \%$, or $100 \%$, respectively, and no more than 1 of the core criteria could worsen by $30 \%$ or more ${ }^{13}$. Pain intensity as judged by the patient (parent) at baseline and last followup were specifically analyzed. The number of children with joint deformities, defined as an irreversible damage to the anatomic structure and/or function that interfered with joint range of motion that could be attributed to the JIA, was also recorded.

Statistics used descriptive analysis for demographics and outcomes using means $\pm \mathrm{SD}$ or medians, as appropriate. Differences between means and medians were evaluated using Student's $t$ and Mann-Whitney U tests, respectively. The level of significance was set at 0.05 .

\section{RESULTS}

Figure 1 illustrates the number of patients included in each category and their outcomes. Table 1 shows the baseline demographics. Forty-three patients were included; 33 were female $(76.7 \%)$. The polyarticular presentation was the most common category, representing 25 of the patients $(58.1 \%)$, with a striking predominance of 23 girls $(92 \%)$ within this category. Among the 10 cases $(23.2 \%)$ with oligoarticular presentation, $3(6.9 \%)$ remained as persistent and $7(16.3 \%)$ turned into a polyarticular form, being classified as extended oligoarticular. Ten $(23.2 \%)$ children were RF-positive, so that $9(20.9 \%)$ of them were classified in the polyarticular category and 1 patient $(2.3 \%)$ was classified as extended oligoarticular. Seven patients (16.3\%) tested positive for the presence of antinuclear antibodies [4 $(9.3 \%)$ polyarticular, 2 $(4.6 \%)$ oligoarticular, and $1(2.3 \%)$ systemic]. 


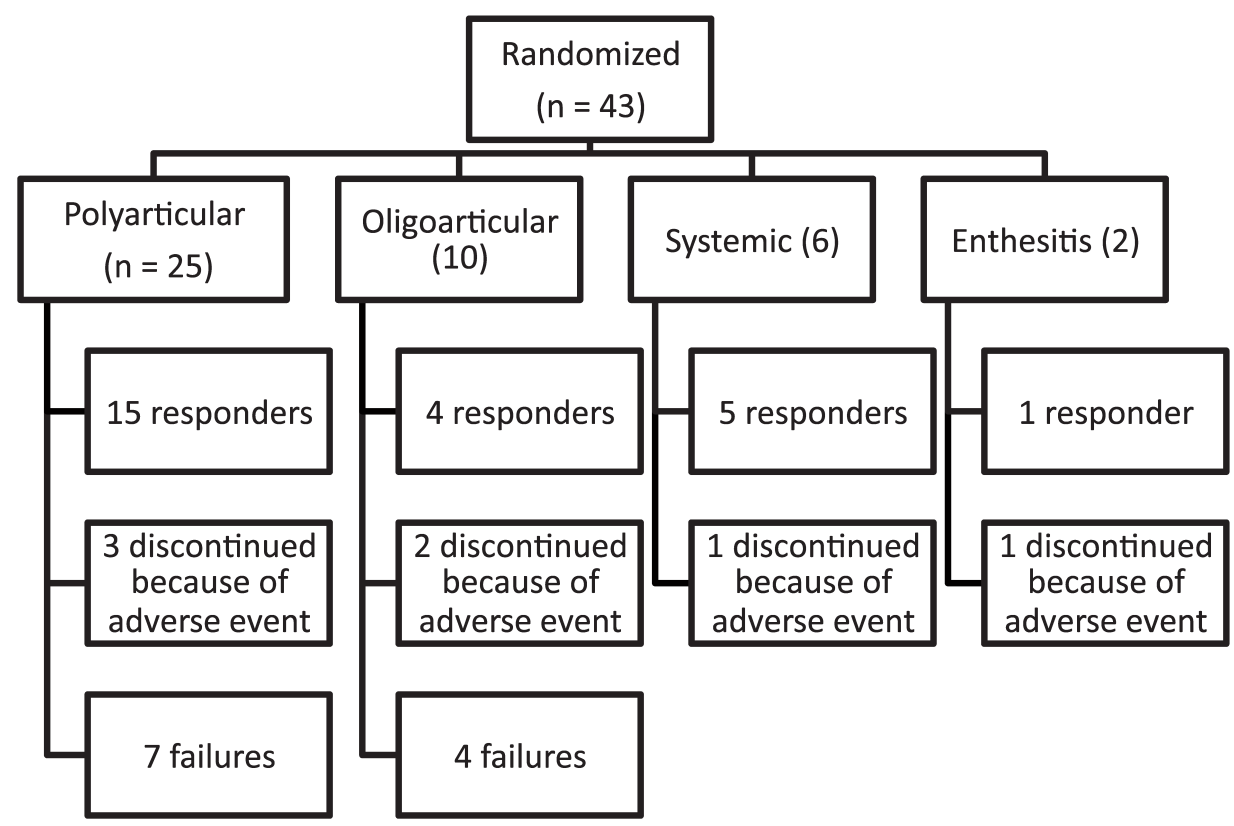

Figure 1. The number of patients with juvenile idiopathic arthritis who were included in each category, and their outcomes.

Table 1. Baseline characteristics of patients with juvenile idiopathic arthritis (JIA). Data are n (\%) unless otherwise indicated.

\begin{tabular}{lc}
\hline Characteristics & \\
\hline Sex & \\
$\quad$ Male & $10(23.3)$ \\
Female & $33(76.7)$ \\
Mean age at onset, yrs, mean \pm SD & $8 \pm 4$ \\
Mean age at diagnosis, yrs, mean \pm SD & $10 \pm 4$ \\
Time between onset of symptoms and definitive & \\
$\quad$ diagnosis, mos, median (range) & $12(1-60)$ \\
Antinuclear antibody positivity & $5(11.6)$ \\
RF positivity & $10(23.2)$ \\
Patients with deformities at time of diagnosis & $10(23.2)$ \\
DAS28, mean \pm SD & $5.58 \pm 0.68$ \\
JIA category & \\
Oligoarticular & $3(7)$ \\
Oligoarticular extended & $7(16)$ \\
Polyarticular RF-negative & $15(35)$ \\
Polyarticular RF-positive & $10(23)$ \\
Enthesitis-related & $2(5)$ \\
Systemic & $6(14)$
\end{tabular}

DAS28: 28-joint Disease Acivity Score; RF: rheumatoid factor.

The mean age at diagnosis was $10 \pm 4$ years, with 32 (74.4\%) being older than 7 years at the time of diagnosis. The age of appearance of first symptoms was $8 \pm 4$ years and the time to definitive diagnosis ranged from 1 to 60 months (median $=12$ mos). Eight patients $(18.6 \%)$ had the diagnosis more than 2 years after the appearance of symptoms.
Ten patients (23.2\%) had existing deformities at the time of diagnosis. Among these, $8(80 \%)$ were in the polyarticular category. The other remaining children in this category were $1(2.3 \%)$ in the enthesitis and the other in the systemic category. Although the difference did not reach statistical significance $(p=0.257)$, it was remarkable that 9 of the 33 girls and 1 of the 10 boys had deformities at the beginning of the protocol. At last followup, those numbers remained unchanged.

Table 2 displays the data concerning the drugs prescribed. All patients used other nonbiologic DMARD before starting LEF, as follows: all 43 used oral MTX; 2 patients $(4.6 \%)$ used sulfasalazine associated with MTX, and $11(25.5 \%)$ used chloroquine diphosphate along with MTX. Twenty-seven patients $(62.7 \%)$ were using oral steroids at the start of the protocol, comprising 15 (34.9\%), $6(13.9 \%), 4(9.3 \%), 1(2.3 \%), 1(2.3 \%)$ in the polyarticular, systemic, extended oligoarticular, persistent oligoarticular, and enthesitis categories, respectively. Thirty-one of the 43 patients had LEF added to MTX whereas 12 had LEF used as monotherapy. The mean daily dose of prednisone was $0.156 \mathrm{mg} / \mathrm{kg}$. None of these patients received any biologic DMARD before starting LEF.

At the start of the protocol, 43 children evaluated were considered as having inadequately controlled disease, with $34(79 \%)$ classified as having severe disease (DAS28 > 5.1). The mean MTX dose prior to changing to or adding LEF was $14.5 \pm 4.5 \mathrm{mg} / \mathrm{m}^{2} /$ week. The mean LEF dose during the protocol was $16.6 \pm 5.2 \mathrm{mg} /$ day $(0.34 \mathrm{mg} / \mathrm{kg} /$ day $)$, and the mean duration of LEF therapy was $3.6 \pm 2.2$ years. 
Table 2. Drugs used before start of leflunomide (LEF) in patients with JIA.

\begin{tabular}{lc}
\hline Drugs & \\
\hline Corticosteroids, n (\%) & $27(62.7)$ \\
DMARD, n (\%) & \\
Oral MTX & $43(100)$ \\
MTX isolated & $30(69.7)$ \\
Chloroquine diphosphate + MTX & $11(25.5)$ \\
Sulfasalazine + MTX & $2(4.6)$ \\
Mean time of MTX therapy, mos (range) & $60(1.2-180)$ \\
Mean MTX dose (mg/week) prior to LEF, mean \pm SD & $14.5 \pm 4.5$ \\
\hline
\end{tabular}

JIA: juvenile idiopathic arthritis; DMARD: disease-modifying antirheumatic drug; MTX: methotrexate.

At the end of followup ( $37 \pm 8.9$ mos), 19 patients (44.2\%) had LEF interrupted. In one case the drug was stopped because the child achieved remission criteria (DAS28 < 2.6) after 2 years of uninterrupted treatment with LEF combined with MTX. That girl continued with MTX (5 $\mathrm{mg} /$ week) after withdrawal of LEF. For 11 patients (25.5\%), LEF was considered to have failed, and 9 among these patients (20.9\%) were taking concomitant MTX. Seven patients $(16.2 \%)$ were considered intolerant of LEF. For 3 of them $(6.9 \%)$ the intolerance was due to nausea and abdominal pain, and $4(9.3 \%)$ had increased liver enzymes ( $>2 \times$ upper limit of normal). Two of the patients with an increase in liver enzymes were taking concomitant MTX $(7.5 \mathrm{mg} /$ week $)+$ LEF $(20 \mathrm{mg} / \mathrm{d})$. After withdrawal of both compounds, liver enzymes returned to normal in 1 month. Two months later, 1 of those latter patients had reinstitution of LEF, which was maintained as the sole DMARD for 39 months until the end of the study without adverse events. There were no other clinically relevant laboratory changes during followup. Our patients were also followed in the pediatric outpatient clinic that gives us support in clinical management. Although the number and type of infections were not individually registered, there were no records of serious infections, no pregnancies, no patient required hospitalization, and there were no deaths.

The efficacy data are summarized in Table 3 . The mean DAS28 of the whole group of 43 patients at the start of the protocol $(5.57 \pm 0.68)$ dropped to $3.7 \pm 1.2$ at the end of followup. At the end of the protocol, 16 patients (37.2\%) had a low DAS28 $(<3.2)$ including $12(27.9 \%)$ taking LEF + MTX and 4 (9.3\%) taking monotherapy with LEF; 18 patients $(41.8 \%)$ had a DAS28 between 3.2 and 5.1 and 9 (20.9\%) had a DAS28 higher than 5.1, indicating severe disease. There was also a significant change in the pain as judged by the patient (parent) at baseline as compared to the last followup, dropping from a mean of $3.79 \pm 1.9$ to $3.65 \pm$ 2.0 ( $\mathrm{p}<0.001$ ), respectively. Baseline mean CHAQ scores also significantly dropped from $0.86 \pm 0.7$ to $0.44 \pm 0.5$ ( $\mathrm{p}<$ 0.001 ) at the end of followup. Although the low numbers when evaluating the clinical outcome according to disease subset do not permit a statistical analysis, patients from all subsets, when evaluated as a group, had a mean improvement at last followup, as compared to baseline. Also, there were dropouts in all groups, as shown in Table 4.

Table 5 shows the proportion of patients achieving ACR-Pedi responses. The majority of the patients [28 $(65.1 \%)]$ achieved at least an ACR-Pedi30, including 12 (27.9\%) who achieved at least an ACR-Pedi50 response. The number of children with joint deformities did not change at the end of followup as compared to baseline.

\section{DISCUSSION}

This is the first study, to our knowledge, on the use of LEF as a second-line effective DMARD therapy on JIA in patients from an equatorial area. Although not being a randomized controlled trial, we conducted a prospective longterm followup study on the use of LEF in JIA in a real-life situation following a clinical protocol with defined outcomes.

As a whole, our data reveal that the clinical response achieved with LEF both as add-on therapy to MTX or monotherapy led to a sustained and significant clinical control in about one-third of those MTX-nonresponsive patients with JIA, with an acceptable safety profile.

We have had access to biologics since 2006, provided regularly by our public service. Our choice of MTX as a first-line DMARD in RA and JIA is based on current guidelines used in $\mathrm{RA}^{4}$ and our longterm good clinical experience with this compound. Although we have to mention the economic influence on our decision to choose LEF as our

Table 3. Change from baseline in clinical and functional variables in patients with juvenile idiopathic arthritis treated with leflunomide (LEF). Patients were considered refractory or intolerant to MTX. Evaluations included the 28-joint Disease Activity Score (DAS28), patient (parent) evaluation of pain in a visual analog scale (VAS; 0-100 mm), and the Childhood Health Assessment Questionnaire (CHAQ). Data represent mean \pm SD

\begin{tabular}{lcccc}
\hline Variable & Baseline & Value at Last Followup & Mean Change After LEF & $* \mathrm{p}$ \\
\hline DAS 28 & $5.57 \pm 0.7$ & $3.74 \pm 1.2$ & $-2.17 \pm 1.39 *$ & $<0.001$ \\
VAS & $6.52 \pm 2.5$ & $3.65 \pm 2.0$ & $-2.87 \pm 0.42 *$ & $<0.001$ \\
CHAQ & $0.86 \pm 0.7$ & $0.44 \pm 0.5$ & $-0.41 \pm 0.39 *$ & $<0.001$ \\
\hline
\end{tabular}

* Using Student's t test. 
Table 4. Change from baseline in clinical and functional variables in 43 patients with juvenile idiopathic arthritis (JIA) who were treated with leflunomide (LEF).

\begin{tabular}{lcccc}
\hline Category & $\mathrm{n}(\%)$ & $\begin{array}{c}\text { Baseline DAS28, } \\
\text { mean } \pm \text { SD }\end{array}$ & $\begin{array}{c}\text { Final DAS28, } \\
\text { mean } \pm \text { SD }\end{array}$ & Discontinued LEF, $\mathrm{n}$ \\
\hline Oligoarticular persistent & $3(7)$ & $5.46 \pm 0.4$ & $4.41 \pm 1.1$ & 2 \\
Oligoarticular extended & $7(16)$ & $5.67 \pm 0.8$ & $4.1 \pm 1.4$ & 4 \\
Polyarticular RF+ & $15(35)$ & $5.46 \pm 0.67$ & $3.4 \pm 0.96$ & 4 \\
Polyarticular RF- & $10(23)$ & $5.73 \pm 0.6$ & $4.12 \pm 1.1$ & 7 \\
Enthesitis-related & $2(5)$ & $5.1 \pm 0.2$ & $3.1 \pm 0.7$ & 1 \\
Systemic & $6(14)$ & $5.71 \pm 0.7$ & $2.69 \pm 1$ & 1 \\
\hline
\end{tabular}

DAS28: 28-joint Disease Activity Score; RF: rheumatoid factor.

Table 5. American College of Rheumatology Pediatric response (ACR-Pedi) in 43 patients with juvenile idiopathic arthritis treated with leflunomide.

\begin{tabular}{lccccc}
\hline Categories & $\mathrm{n}$ & ACR-Pedi $<30$ & ACR-Pedi30 & ACR-Pedi50 & ACR-Pedi70 \\
\hline Systemic & 6 & 1 & 1 & 3 & 1 \\
Polyarticular RF+ & 10 & 2 & 6 & 1 & 1 \\
Polyarticular RF- & 15 & 6 & 7 & 1 & 1 \\
Oligoarticular persistent & 3 & 2 & 0 & 1 & 0 \\
Oligoarticular extended & 7 & 3 & 2 & 1 & 1 \\
Enthesitis-related & 2 & 1 & 0 & 1 & 0 \\
Total & 43 & 15 & 16 & 8 & 4 \\
\hline
\end{tabular}

RF: rheumatoid factor.

second-choice DMARD, the main reasons to postpone the introduction of biologic DMARD were safety issues related to our high prevalence of infectious and parasitic diseases such as tuberculosis, hanseniasis, and visceral leishmaniasis.

A previous open-label study of patients with JIA refractory to MTX and a double-blinded trial comparing MTX to LEF also reported an acceptable safety profile with significant improvement in signs and symptoms ${ }^{5}$. However, worldwide experience and/or recommendations regarding JIA treatment, including the specific issue of LEF use, are yet to be defined. More recently, following a systematic literature review, the ACR did not recommend the routine use of LEF as a preferable second-choice DMARD option in patients with JIA, as we currently adopted in our service. As a whole, the recommendations were that MTX was generally preferable as the first-choice DMARD, followed by a biologic agent in the case of refractoriness or intolerance to $\mathrm{MTX}^{14}$.

Current treatment outcomes and evaluation in JIA have greatly improved ${ }^{9}$. However, by the time we designed our protocol, there were no specific instruments to establish outcomes in JIA. Our strategy was to define a target of low disease activity or remission using the DAS28 score to record the patient (parent) opinion concerning disease activity status. Although this strategy is open to debate, a study has shown that the RA DAS28 scores performed similarly to the Juvenile Arthritis Disease Activity Score in patients with JIA ${ }^{15}$. Trying to define goals as a treat-to-target approach, our patients were prospectively and closely monitored so that each patient that was not considered in low disease activity or remission according to DAS28 score was seen monthly. Those who did not achieve the outcome in 6 months and used combined MTX and LEF were considered nonresponsive to treatment; LEF was discontinued and a biologic was then started. For those taking LEF monotherapy, a biologic could then be added.

The sustained good clinical response was also a very positive result in our cohort. After a mean period of more than 3 years of followup for the whole cohort, the number of patients with high disease activity dropped from 39 to 9 . Therefore, the addition of LEF to these MTX-nonresponsive patients with JIA led to a relevant improvement in two-thirds of those with high disease activity. This is also reflected by the significant reduction in the pain component as judged by the patient (parent). Considering that CHAQ scores also significantly improved, we can also infer that functional impairment was lessened in these patients after the introduction of LEF. Moreover, the number of joints with deformities did not increase, even in those cases that did not achieve the defined outcome of low disease activity. It should be mentioned that at study entry, patients in the systemic category had only joint features.

An ACR-Pedi30 response was achieved by 28 of our patients $(65 \%)$. A previous longterm open-label trial with LEF in polyarticular patients with JIA produced a similar response. However, a response greater than ACR-Pedi50

Personal non-commercial use only. The Journal of Rheumatology Copyright @ 2014. All rights reserved. 
was seen in only $29.7 \%$ of our patients, as compared to $47 \%$ in that trial $^{5}$. We cannot exclude the possibility that persistence with LEF could have resulted in further improvement in those who achieved a partial response in the first 6 months.

Notwithstanding the clinical response, the safety profile was excellent. It has to be stressed that most of our patients used LEF concomitant to MTX and that only 6 of them, regardless of whether they used LEF as an add-on or monotherapy, had to definitively discontinue LEF because of adverse events. In addition, we had no hospitalizations or deaths during the protocol. Laboratory alterations were also not relevant, meaning that there were no significant changes in blood cell counts or routine biochemistry. Liver enzymes elevations were mild and led to definitive discontinuation in only 3 of the 43 exposed patients. As mentioned, those patients were under close surveillance both by a pediatrician and a rheumatologist. Therefore, the possibility of unchecked serious infections, malignancies, or other serious adverse events was low. What probably accounted for these safety data were avoiding the use of an LEF loading dose, adherence to dosage using a previously suggested protocol ${ }^{5}$ and close surveillance, and using the treat-to-target monthly approach to aim for low disease activity. We had no pregnancies during the study period. However, it is necessary to collect longterm data on our patients who received LEF, with special attention to girls, aiming to detect a possible negative effect on pregnancy outcomes in the future.

Biologic therapy is a mainstay in the treatment of RA and also in patients with JIA refractory to $\mathrm{MTX}^{1,3,4}$, providing an ACR-Pedi30 response in clinical trials over $70 \% 16,17$. A trial using an antiinterleukin 6 antibody in patients with systemic JIA reported that $80 \%$ achieved at least $70 \%$ improvement ${ }^{17}$. Therefore, our results with LEF seem modest, especially for a higher ACR-Pedi response. However, a possible increased risk of the incidence of adverse events, with special concern about infections, malignancies, and induction of autoimmune phenomena, still requires attention when using biologics $16,17,18$.

Using DAS28 can be seen as a limitation of our study, as discussed. Being an open-label trial may also have influenced clinical evaluation. One major limitation, however, could be achievement of an ambitious primary outcome in the first 6 months. This strategy may have prematurely excluded patients with a partial response to LEF who could have improved later. However, current experience with LEF in JIA is scant. Therefore, despite limitations, our data reflect a real-life experience, adding support to an acceptable safety profile with the longterm use of LEF, under close scrutiny, in patients with JIA.

This is the first report, to our knowledge, on the prospective evaluation of LEF use in patients with an equatorial nonwhite ancestry. Moreover, to our knowledge, this is the largest and longest clinical protocol of LEF use in JIA. In addition to showing a statistically significant response, based on the baseline and final DAS28 scores, we believe that our results are clinically relevant. The reasons for this are both the improvement obtained and a documentation of an acceptable safety profile. When evaluating our experience (including our unpublished observations) with the treatment of patients with JIA, after combining the number of patients achieving a low DAS28 or clinical remission, our conservative estimate is that around two-thirds of our patients have a clinically manageable disease with the use of LEF, either alone or combined with MTX and low-dose steroids. LEF is an effective and safe option that appears worthwhile for patients with JIA who are considered unresponsive or intolerant of MTX prior to starting biologics.

\section{REFERENCES}

1. Kemper AR, van Mater HA, Coeytaux RR, Williams Jr JW, Sanders GD. Systematic review of disease-modifying antirheumatic drugs for juvenile idiopathic arthritis. BMC Pediatr 2012;12:29.

2. Behrens F, Koehm M, Burkhardt H. Update 2011: leflunomide in rheumatoid arthritis - strengths and weaknesses. Curr Opin Rheumatol 2011;23:282-7.

3. Furst DE, Keystone EC, Braun J, Breedveld FC, Burmester GR, De Benedetti F, et al. Updated consensus statement on biological agents for the treatment of rheumatic diseases, 2011. Ann Rheum Dis 2012;71:i2-45.

4. Pereira IA, Mota LM, Cruz BA, Brenol CV, Fronza LS, Bertolo MB, et al. 2012 Brazilian Society of Rheumatology Consensus on the management of comorbidities in patients with rheumatoid arthritis. Rev Bras Reumatol 2012;52:474-95.

5. Silverman E, Mouy R, Spiegel L, Jung LK, Saurenmann RK, Lahdenne P, et al. Leflunomide or methotrexate for juvenile rheumatoid arthritis. N Engl J Med 2005;352:1655-66.

6. Foeldvari I, Wierk A. Effectiveness of leflunomide in patients with juvenile idiopathic arthritis in clinical practice. J Rheumatol 2010;37:1763-7.

7. Ellis JA, Munro JE, Ponsonby AL. Possible environmental determinants of juvenile idiopathic arthritis. Rheumatology 2010;49:411-25.

8. Grigor C, Capell H, Stirling A, McMahon AD, Lock P, Vallance R, et al. Effect of a treatment strategy of tight control for rheumatoid arthritis (the TICORA study): a single-blind randomised controlled trial. Lancet 2004;364:263-9.

9. Consolaro A, Negro G, Lanni S, Solari N, Martini A, Ravelli A. Toward a treat-to-target approach in the management of juvenile idiopathic arthritis. Clin Exp Rheumatol 2012;30 Suppl 73:S157-62.

10. Magnani A, Pistorio A, Magni-Manzoni S, Falcone A, Lombardini $\mathrm{G}$, Bandeira M, et al. Achievement of a state of inactive disease at least once in the first 5 years predicts better outcome of patients with polyarticular juvenile idiopathic arthritis. J Rheumatol 2009;36:628-34

11. Prevoo ML, van 't Hof MA, Kuper HH, van Leeuwen MA, van de Putte LB, van Riel PL. Modified disease activity scores that include twenty-eight-joint counts. Development and validation in a prospective longitudinal study of patients with rheumatoid arthritis. Arthritis Rheum 1995;38:44-8.

12. Petty RE, Southwood TR, Manners P, Baum J, Glass DN, Goldenberg $\mathrm{J}$, et al. International League of Associations for Rheumatology classification of juvenile idiopathic arthritis: second revision, Edmonton, 2001. J Rheumatol 2004;31:390-2.

Personal non-commercial use only. The Journal of Rheumatology Copyright $\subset$ 2014. All rights reserved. 
13. Giannini EH, Ruperto N, Ravelli A, Lovell DJ, Felson DT, Martini A. Preliminary definition of improvement in juvenile arthritis. Arthritis Rheum 1997;40:1202-9.

14. Beukelman T, Patkar NM, Saag KG, Tolleson-Rinehart S, Cron RQ, DeWitt EM. 2011 American College of Rheumatology recommendations for the treatment of juvenile idiopathic arthritis: initiation and safety monitoring of therapeutic agents for the treatment of arthritis and systemic features. Arthritis Care Res 2011;63:465-82.

15. Ringold S, Bittner R, Neogi T, Wallace CA, Singer NG. Performance of rheumatoid arthritis disease activity measures and juvenile arthritis disease activity scores in polyarticular-course juvenile idiopathic arthritis: analysis of their ability to classify the
American College of Rheumatology pediatric measures of response and the preliminary criteria for flare and inactive disease. Arthritis Care Res 2010;62:1095-102.

16. Ruperto N, Lovell DJ, Cuttica R, Wilkinson N, Woo P, Espada G, et al. A randomized, placebo-controlled trial of infliximab plus methotrexate for the treatment of polyarticular-course juvenile rheumatoid arthritis. Arthritis Rheum 2007;56:3096-106.

17. Ruperto N, Lovell DJ, Quartier P, Paz E, Rubio-Pérez N, Silva CA, et al. Long-term safety and efficacy of abatacept in children with juvenile idiopathic arthritis. Arthritis Rheum 2010;62:1792-802.

18. De Benedetti F, Brunner HI, Ruperto N, Kenwright A, Wright S, Calvo I, et al. Randomized trial of tocilizumab in systemic juvenile idiopathic arthritis. N Engl J Med 2012;367:2385-95. 